\title{
On the Existence of Infinitely Many Solutions for Nonlocal Systems with Critical Exponents
}

\author{
M. Khiddi ${ }^{1}$ and R. Echarghaoui ${ }^{2}$ \\ ${ }^{1}$ E.G.A.L, Département de Mathématiques, Faculté des Sciences, Université Ibn Tofail, BP 133, Kénitra, Morocco \\ ${ }^{2}$ L.A.G.A, Département de Mathématiques, Faculté des Sciences, Université Ibn Tofail, BP 133, Kénitra, Morocco \\ Correspondence should be addressed to M. Khiddi; mostapha-2@hotmail.com
}

Received 10 March 2016; Accepted 29 May 2016

Academic Editor: Shuangjie Peng

Copyright (c) 2016 M. Khiddi and R. Echarghaoui. This is an open access article distributed under the Creative Commons Attribution License, which permits unrestricted use, distribution, and reproduction in any medium, provided the original work is properly cited.

\begin{abstract}
We study a class of semilinear nonlocal elliptic systems posed on settings without compact Sobolev embedding. By employing critical point theory and concentration estimates, we prove the existence of infinitely many solutions for values of the dimension $N$, where $N>6 s$, provided $0<s<1$.
\end{abstract}

\section{Introduction and Main Result}

Nonlocal problems and operators have enjoyed much attention from mathematicians in recent years probably due to their interesting analytical structure and their numerous physical applications in many fields such as flame propagation, conservation laws, ultrarelativistic limits of quantum mechanics, quasi-geostrophic flows, and the thin obstacle problem (see [1-3]). In this paper, we are concerned with the study of the infinitely many solutions for the following system:

$$
\begin{aligned}
& (-\Delta)^{s} u=\frac{2 \alpha}{\alpha+\beta}|u|^{\alpha-2} u|v|^{\beta}+\lambda u \quad \text { in } \Omega, \\
& (-\Delta)^{s} v=\frac{2 \beta}{\alpha+\beta}|u|^{\alpha}|v|^{\beta-2} v+\mu v \quad \text { in } \Omega \text {, } \\
& u=v=0 \quad \text { on } \partial \Omega \text {, }
\end{aligned}
$$

where $\Omega$ is an open bounded domain in $\mathbb{R}^{N}, \lambda, \mu>0$ and $\alpha>1, \beta>1$ satisfy $\alpha+\beta=2_{s}^{*}=2 N /(N-2 s), s \in(0,1)$ and $N>2 s$. When $\alpha=\beta, \lambda=\mu$, and $u=v$, problem (1) reduces to the Brézis-Nirenberg type problem with fractional Laplacian:

$$
\begin{aligned}
(-\Delta)^{s} u & =|u|^{2_{s}^{*}-2} u+\lambda u \quad \text { in } \Omega, \\
u & =0 \quad \text { on } \partial \Omega .
\end{aligned}
$$

In [4], Brézis and Nirenberg considered the existence of positive solutions for problem (2) with $s=1$. Such a problem involves the critical Sobolev exponent $2^{*}=2 N /(N-2)$ for $N \geq 3$, and it is well known that the Sobolev embedding $H_{0}^{1}(\Omega) \hookrightarrow L^{2^{*}}(\Omega)$ is not compact even if $\Omega$ is bounded. Hence, the associated functional of problem (2) does not satisfy the Palais-Smale condition, and critical point theory can not be applied directly to find solutions of the problem. However, it is found in [4] that the functional satisfies the $(\mathrm{PS})_{c}$ condition for $c \in\left(0,(1 / N) S^{N / 2}\right)$, where $S$ is the best Sobolev constant and $(1 / N) S^{N / 2}$ is the least energy level at which the Palais-Smale condition fails. So a positive solution can be found if the mountain pass value corresponding to problem (2) is strictly less than $(1 / N) S^{N / 2}$. In [5], a concentration-compactness principle was developed to treat noncompact critical variational problems. In the study of the existence of multiple solutions for critical problems, to retain 
the compactness, it is necessary to have a full description of energy levels at which the associated functional does not satisfy the Palais-Smale condition. A global compactness result is found in [6], which describes precisely the obstacles of the compactness for critical semilinear elliptic problems. This compactness result shows that above a certain energy level it is impossible to prove the Palais-Smale condition. For this reason, to obtain infinitely many solutions for the critical problem, it is essential to find a condition that can replace the standard Palais-Smale condition. In [7], Devillanova and Solimini considered (2) with $s=1$ and obtained infinitely many solutions for every $\lambda>0$ if $N \geq 7$. They proved this latter result by employing the concentration estimates and lower bound of augmented Morse index on min-max points (see [8]), which seem unapplicable to the case of (1) directly. This work was extended to an analogous problem involving $p$-Laplacian for $1<p<\infty$ by Cao et al. [9]. They proved that if $N>p^{2}+p$, the following problem,

$$
-\Delta_{p} u=|u|^{p^{*}-2} u+\lambda|u|^{p-2} u \quad \text { in } \Omega, u=0 \text { on } \partial \Omega \text {, }
$$

where $\mu>0$ and $p^{*}=p N /(N-p)$, has infinitely many solutions. Recently, Yan et al. extended the result in [10] to problem (2) and obtained infinitely many solutions for every $\lambda>0$ if $N>6 s$, where $0<s<1$. Similar problems to (1) for the Laplacian operator have been studied extensively in recent years; see $[6,11-14]$ and the references therein. In particular, Alves et al. [11] studied the $p$-Laplacian system with critical growth and obtained the existence of infinitely many solutions if $N>p^{2}+p$. An important cornerstone for these works has been laid out in a remarkable paper [15], where Caffarelli and Silvestre gave a new formulation of the fractional Laplacian through Dirichlet-Neumann maps. This is extensively used in the recent literature since it allows transforming nonlocal problems to local ones, which permits the use of variational methods. This will come in handy for this work. A more general form of the fractional operator has been studied and a multiplicity of solutions has been shown in several cases. For further reading, we refer the reader to these recently published papers on the fractional Laplacian [16-22].

In this paper, we prove that (1) has infinitely many solutions under the following conditions: $N>6 s$, where $0<s<1$, by studying system (1) in the critical case $\alpha+\beta=2_{s}^{*}$. We adapt the original idea used in [7] which was described in the above paragraph (see also [9-11, 13]). After perturbing problem (1) into a subcritical case with a (PS) functional in all energy levels, estimates on the set of the solution sequences to the subcritical case of (1), a global compactness argument, and a (local) Pohozaev identity are used to establish the strong convergence and finally use min-max theorems on a genus homotopic class to produce infinitely many critical values. The paper is organized as follows: in Section 2, some notations and preliminary results are established. In Section 3, we establish a local Pohozaev identity which allows us to prove the $E_{0}^{s}\left(\mathscr{C}_{\Omega}\right)$-strong convergence of solutions for the subcritical case of problem (18), and, finally, we show how this technique allows the application of classical min-max arguments to (1) and to prove, in this way, the existence of infinitely many solutions. Our main result is the following.

Theorem 1. Let $0<s<1$ and $\lambda \geq 0, \mu \geq 0$ with $\lambda+\mu>0$. Then problem (1) has infinitely many solutions when $N>6$ s.

\section{Notation and Preliminaries}

The powers $(-\Delta)^{s}$ of the positive Laplacian operator $-\Delta$, in $\Omega$, with zero Dirichlet boundary conditions are defined via its spectral decomposition, namely,

$$
(-\Delta)^{s} u(x):=\sum_{j=1}^{k} a_{j} \rho_{j}^{s} \varphi_{j}(x),
$$

where $\left(\rho_{j}, \varphi_{j}\right)$ is the sequence of eigenvalues and eigenfunctions of the operator $(-\Delta)$ in $\Omega$ under zero Dirichlet boundary data and $a_{j}$ are the coefficients of $u$ for the base $\left\{\varphi_{j}\right\}_{j=1}^{\infty}$ in $L^{2}(\Omega)$. In fact, the fractional Laplacian $(-\Delta)^{s}$ is well defined in the space of functions

$$
\begin{gathered}
H_{0}^{s}(\Omega):=\left\{u=\sum_{j=1}^{k} a_{j} \varphi_{j} \in L^{2}(\Omega):\|u\|_{H_{0}^{s}}\right. \\
\left.=\left(\sum_{j=1}^{k} a_{j}^{2} \rho_{j}^{s}\right)^{1 / 2}<\infty\right\},
\end{gathered}
$$

and $\|u\|_{H_{0}^{s}}=\left\|(-\Delta)^{s / 2} u\right\|_{L^{2}(\Omega)}$.

A pair of functions $(u, v) \in H_{0}^{s}(\Omega) \times H_{0}^{s}(\Omega)$ is said to be a weak solution of problem (1) if

$$
\begin{aligned}
& \int_{\Omega}\left((-\Delta)^{s / 2} u(-\Delta)^{s / 2} \varphi_{1}+(-\Delta)^{s / 2} v(-\Delta)^{s / 2} \varphi_{2}\right) d x \\
& -\int_{\Omega}\left(\lambda u \varphi_{1}+\mu v \varphi_{2}\right) d x \\
& \quad-\frac{2 \alpha}{\alpha+\beta} \int_{\Omega}|u|^{\alpha-2} u|v|^{\beta} \varphi_{1} d x \\
& -\frac{2 \beta}{\alpha+\beta} \int_{\Omega}|u|^{\alpha}|v|^{\beta-2} v \varphi_{2} d x=0,
\end{aligned}
$$

for all $\left(\varphi_{1}, \varphi_{2}\right) \in H_{0}^{s}(\Omega) \times H_{0}^{s}(\Omega)$. Solutions to problem (1) will be obtained as critical points of the corresponding energy functional

$$
\begin{aligned}
J_{\lambda, \mu}(u, v):= & \frac{1}{2} \int_{\Omega}\left(\left|(-\Delta)^{s / 2} u\right|^{2}+\left|(-\Delta)^{s / 2} v\right|^{2}\right) d x \\
& -\frac{1}{2} \int_{\Omega}\left(\lambda|u|^{2}+\mu|v|^{2}\right) d x \\
& -\frac{2}{\alpha+\beta} \int_{\Omega}|u|^{\alpha}|v|^{\beta} d x .
\end{aligned}
$$

The functional is well defined in $H_{0}^{s}(\Omega) \times H_{0}^{s}(\Omega)$, and, moreover, the critical points of the functional $J_{\lambda, \mu}$ correspond to solutions of (1). We now conclude the main ingredients 
of a recently developed technique use in order to deal with fractional powers of the Laplacian operator. Motivated by the work of Caffarelli and Silvestre [15], several authors have considered an equivalent definition of the operator $(-\Delta)^{s / 2}$ in a bounded domain with zero Dirichlet boundary data means of auxiliary variable, see $[2,23]$. Associated with the bounded domain $\Omega$, let us consider the cylinder $\mathscr{C}_{\Omega}:=\Omega \times(0,+\infty) \subset$ $\mathbb{R}_{+}^{N+1}$. Now, for a function $u \in H_{0}^{s}$, we define the $s$-harmonic extension $w=E_{s}(u)$ to the cylinder $\mathscr{C}_{\Omega}$ as the solution of the problem:

$$
\begin{aligned}
\operatorname{div}\left(y^{1-2 s} \nabla w\right) & =0 \quad \text { in } \mathscr{C}_{\Omega} \\
w & =0 \quad \text { on } \partial_{L} \Omega \\
w & =u \quad \text { on } \Omega \times\{0\}, \\
(-\Delta)^{s} u(x) & =-k_{s} \lim _{y \rightarrow 0^{+}} y^{1-2 s} \frac{\partial w}{\partial y}(x, y),
\end{aligned}
$$

where $k_{s}=2^{1-2 s} \Gamma(1-s) / \Gamma(s)$ is a normalization constant. The extension function $w(x, y)$ belongs to the space

$$
\begin{aligned}
& H_{0, L}^{1}\left(\mathscr{C}_{\Omega}\right):=\left\{w \in L^{2}\left(\mathscr{C}_{\Omega}\right): w\right. \\
& \left.\quad=0 \text { on } \partial_{L} \Omega, \int_{\mathscr{C}_{\Omega}} y^{1-2 s}|\nabla w|^{2} d x d y<\infty\right\}
\end{aligned}
$$

endowed with the norm

$$
\|z\|_{H_{0, L}^{1}\left(\mathscr{C}_{\Omega}\right)}:=\left(\int_{\mathscr{C}_{\Omega}} y^{1-2 s}|\nabla z|^{2} d x d y\right)^{1 / 2} .
$$

The extension operator is an isometry between $H_{0}^{s}(\Omega)$ and $H_{0, L}^{1}\left(\mathscr{C}_{\Omega}\right)$; namely,

$$
\|u\|_{H_{0}^{s}(\Omega)}=\left\|E_{s}(u)\right\|_{H_{0, L}^{1}\left(\mathscr{C}_{\Omega}\right)}, \quad \forall u \in H_{0}^{s}(\Omega) .
$$

With this extension, we can reformulate (1) as the following local problem:

$$
\begin{aligned}
& -\operatorname{div}\left(y^{1-2 s} \nabla w_{1}\right)=0, \\
& -\operatorname{div}\left(y^{1-2 s} \nabla w_{2}\right)=0 \\
& w_{1}=w_{2}=0, \quad \text { on } \partial_{L} \Omega, \\
& y^{1-2 s} \frac{\partial w_{1}}{\partial \nu} \\
& =\frac{2 \alpha}{\alpha+\beta}\left|w_{1}(x, 0)\right|^{\alpha-2} w_{1}(x, 0)\left|w_{2}(x, 0)\right|^{\beta} \\
& \quad+\lambda w_{1}(x, 0) \quad \text { on } \Omega \times\{0\}, \\
& y^{1-2 s} \frac{\partial w_{2}}{\partial \nu} \quad \text { on } \Omega \times\{0\}, \\
& =\frac{2 \beta}{\alpha+\beta}\left|w_{1}(x, 0)\right|^{\alpha}\left|w_{2}(x, 0)\right|^{\beta-2} w_{2}(x, 0) \\
& +\mu w_{2}(x, 0) \quad
\end{aligned}
$$

in $\mathscr{C}_{\Omega}$, where

$$
\frac{\partial w_{i}}{\partial \nu}:=-k_{s} \lim _{y \rightarrow 0^{+}} y^{1-2 s} \frac{\partial w_{i}}{\partial y}, \quad i=1,2
$$

and $w_{1}, w_{2} \in H_{0, L}^{1}\left(\mathscr{C}_{\Omega}\right)$ are the $s$-harmonic extension of $u, v \in$ $H_{0}^{s}(\Omega)$, respectively. Let

$$
E_{0}^{s}\left(\mathscr{C}_{\Omega}\right):=H_{0, L}^{1}\left(\mathscr{C}_{\Omega}\right) \times H_{0, L}^{1}\left(\mathscr{C}_{\Omega}\right)
$$

be equipped with the norm

$$
\left\|\left(w_{1}, w_{2}\right)\right\|_{E_{0}^{s}\left(\mathscr{C}_{\Omega}\right)}=\left\|w_{1}\right\|_{H_{0, L}^{1}\left(\mathscr{C}_{\Omega}\right)}+\left\|w_{2}\right\|_{H_{0, L}^{1}\left(\mathscr{C}_{\Omega}\right)} .
$$

An energy solution to this problem is a function $\left(w_{1}, w_{2}\right) \in$ $E_{0}^{\mathcal{s}}\left(\mathscr{C}_{\Omega}\right)$ satisfying

$$
\begin{aligned}
\int_{\mathscr{C}_{\Omega}} & y^{1-2 s} \nabla w_{1} \nabla \varphi_{1} d x d y+\int_{\mathscr{C}_{\Omega}} y^{1-2 s} \nabla w_{2} \nabla \varphi_{2} d x d y \\
= & \lambda \int_{\Omega} w_{1} \varphi_{1} d x+\frac{2 \alpha}{\alpha+\beta} \int_{\Omega}\left|w_{1}\right|^{\alpha-2} w_{1}\left|w_{2}\right|^{\beta} \varphi_{1} d x \\
& +\mu \int_{\Omega} w_{2} \varphi_{2} d x \\
& +\frac{2 \beta}{\alpha+\beta} \int_{\Omega}\left|w_{1}\right|^{\alpha}\left|w_{2}\right|^{\beta-2} w_{2} \varphi_{2} d x
\end{aligned}
$$

for all $\left(\varphi_{1}, \varphi_{2}\right) \in E_{0}^{s}\left(\mathscr{C}_{\Omega}\right)$. If $\left(w_{1}, w_{2}\right) \in E_{0}^{s}\left(\mathscr{C}_{\Omega}\right)$ satisfies (12), then $(u, v)=\left(w_{1}(\cdot, 0), w_{2}(\cdot, 0)\right)$, defined in the sense of traces, belongs to the space $H_{0}^{s}(\Omega) \times H_{0}^{s}(\Omega)$ and it is a solution of the original problem (1). The associated energy functional to problem (12) is denoted by

$$
\begin{aligned}
I_{\lambda, \mu}(w):= & I_{\lambda, \mu}\left(w_{1}, w_{2}\right) \\
= & \frac{1}{2} \int_{\mathscr{C}_{\Omega}} y^{1-2 s}\left(\left|\nabla w_{1}\right|^{2}+\left|\nabla w_{2}\right|^{2}\right) d x d y \\
& -\frac{1}{2} \int_{\Omega}\left(\lambda\left|w_{1}\right|^{2}+\mu\left|w_{2}\right|^{2}\right) d x \\
& -\frac{2}{\alpha+\beta} \int_{\Omega}\left|w_{1}\right|^{\alpha}\left|w_{2}\right|^{\beta} d x .
\end{aligned}
$$

Critical points of $I_{\lambda, \mu}$ in $E_{0}^{s}\left(\mathscr{C}_{\Omega}\right)$ correspond to the critical points of $J_{\lambda, \mu}: H_{0}^{s}(\Omega) \times H_{0}^{s}(\Omega) \rightarrow \mathbb{R}$. Since the problem is critical, the functional $I_{\lambda, \mu}$ does not satisfy the Palais-Smale condition. Thus the min-max theorems can not be applied directly to obtain infinitely many solutions for (1). Following 
the original idea in [7], employed in a closer setting in [10], we deal first with the following perturbed problem:

$$
\begin{aligned}
& -\operatorname{div}\left(y^{1-2 s} \nabla w_{1}\right)=0, \\
& -\operatorname{div}\left(y^{1-2 s} \nabla w_{2}\right)=0 \\
& w_{1}=w_{2}=0, \quad \text { on } \partial_{L} \Omega \\
& y^{1-2 s} \frac{\partial \omega_{1}}{\partial \nu} \quad \text { in } \mathscr{C}_{\Omega} \\
& =\frac{2(\alpha-\varepsilon)}{\alpha+\beta-2 \varepsilon}\left|w_{1}(x, 0)\right|^{\alpha-2-\varepsilon} w_{1}(x, 0)\left|w_{2}(x, 0)\right|^{\beta-\varepsilon} \\
& \quad+\lambda w_{1}(x, 0) \quad \text { on } \Omega \times\{0\} \\
& y^{1-2 s} \frac{\partial \omega_{2}}{\partial \nu} \\
& =\frac{2(\beta-\varepsilon)}{\alpha+\beta-2 \varepsilon}\left|w_{1}(x, 0)\right|^{\alpha-\varepsilon}\left|w_{2}(x, 0)\right|^{\beta-2-\varepsilon} w_{2}(x, 0) \\
& +\mu w_{2}(x, 0) \quad \text { on } \Omega \times\{0\},
\end{aligned}
$$

where $\left(w_{1}, w_{2}\right) \in E_{0}^{s}\left(\mathscr{C}_{\Omega}\right)$ and $0<\varepsilon<\min \left\{\alpha-1, \beta-1,2_{s}^{*}-1\right\}$.

The functional corresponding to (18) becomes

$$
\begin{aligned}
I_{\lambda, \mu}^{\varepsilon}(w, \varphi)= & \frac{1}{2} \int_{\mathscr{C}_{\Omega}} y^{1-2 s}\left(|\nabla w|^{2}+|\nabla \varphi|^{2}\right) d x d y \\
& -\frac{1}{2} \int_{\Omega}\left(\lambda|w|^{2}+\mu|\varphi|^{2}\right) d x \\
& -\frac{2}{\alpha+\beta-2 \varepsilon} \int_{\Omega}|w|^{\alpha-\varepsilon}|\varphi|^{\beta-\varepsilon} d x,
\end{aligned}
$$

where $(w, \varphi) \in E_{0}^{\mathcal{s}}\left(\mathscr{C}_{\Omega}\right)$. Now $I_{\lambda, \mu}^{\varepsilon}$ is even and satisfies the Palais-Smale condition at all energy levels. It follows from the symmetric mountain pass lemma [24] that (18) has infinitely many solutions. More precisely, it follows from [25, Theorem 6.1] that there are positive numbers $c_{\varepsilon, l}, l=1,2, \ldots$, with $c_{\varepsilon, l} \rightarrow+\infty$, as $l \rightarrow+\infty$ and a solution $\left(w_{\varepsilon, l}, \varphi_{\varepsilon, l}\right)$ for (18), satisfying $I^{\varepsilon}\left(w_{\varepsilon, l}, \varphi_{\varepsilon, l}\right)=c_{\varepsilon, l}$. To obtain the existence of infinitely many solutions for (1), the first step is to investigate whether $\left(w_{\varepsilon, l}, \varphi_{\varepsilon, l}\right)$ converges strongly in $E_{0}^{\mathcal{S}}\left(\mathscr{C}_{\Omega}\right)$ as $\varepsilon \rightarrow$ 0 . That is, we need to study the compactness of the set of solutions for (18), with $\varepsilon>0$ small enough.

\section{3. $E_{0}^{s}\left(\mathscr{C}_{\Omega}\right)$-Strong Convergence of Solutions for the Subcritical Case of Problem (18)}

In this section, we establish the strong convergence of solutions for the perturbed problem (18). Throughout this paper, we denote the norms of $E_{0}^{s}\left(\mathscr{C}_{\Omega}\right), L^{l}(\Omega)(1 \leq l<\infty)$ by $\left\|\left(w_{1}, w_{2}\right)\right\|_{E_{0}^{s}\left(\mathscr{C}_{\Omega}\right)}=\left\|w_{1}\right\|_{H_{0, L}^{1}\left(\mathscr{C}_{\Omega}\right)}+\left\|w_{2}\right\|_{H_{0, L}^{1}\left(\mathscr{C}_{\Omega}\right)},\|u\|_{L^{l}(\Omega)}=$ $\left(\int_{\Omega}|u|^{l} d x\right)^{1 / l}$, respectively, and positive constants (possibly different) by $C$.
Proposition 2. Suppose that $N>6 s$ with $0<s<1$ and $\lambda, \mu \geq 0$ satisfy $\lambda+\mu>0$. Then for any sequence $\left(u_{n}, v_{n}\right)(n=$ $1,2, \ldots)$ which is a solution of (18) with $\varepsilon=\varepsilon_{n} \rightarrow 0$, and for $\left(u_{n}, v_{n}\right)$ satisfies $\left\|\left(u_{n}, v_{n}\right)\right\|_{E_{0}^{s}\left(\mathscr{C}_{\Omega}\right)} \leq C$ for some constant $C>$ 0 independent of $n$, then $\left(u_{n}, v_{n}\right)_{n \geq 1}$ has a subsequence which converges strongly in $E_{0}^{s}\left(\mathscr{C}_{\Omega}\right)$ as $n \rightarrow+\infty$.

Before giving the proof of Proposition 2, we give some estimates for $\left(u_{n}, v_{n}\right)$. Let $D$ be a bounded domain such that $\Omega \Subset D$ and $\left(u_{n}(x, 0), v_{n}(x, 0)\right)=(0,0)$ in $D \backslash \Omega$. We choose $A>0$ large enough so that, for all $x, y \in \mathbb{R}$, the following is verified:

$$
\begin{aligned}
& \left.\left|\frac{2 \alpha_{n}}{\alpha_{n}+\beta_{n}}\right| x\right|^{\alpha_{n}-2} x|y|^{\beta_{n}}+\lambda x \mid \leq 2(|x|+|y|)^{2_{s}^{*}-1}+A, \\
& \left.\left|\frac{2 \beta_{n}}{\alpha_{n}+\beta_{n}}\right| x\right|^{\alpha_{n}}|y|^{\beta_{n}-2} y+\mu y \mid \leq 2(|x|+|y|)^{2_{s}^{*}-1}+A,
\end{aligned}
$$

for all $x, y \in \mathbb{R}$. Let $\left(\omega_{n}\right)(n=1,2, \ldots)$ with $\omega_{n} \geq 0$ be a solution of

$$
\begin{aligned}
&-\operatorname{div}\left(y^{1-2 s} \nabla \omega\right)=0, \quad \text { in } \mathscr{C}_{D}, \\
& \omega=0, \quad \text { on } \partial_{L} D, \\
& y^{1-2 s} \frac{\partial \omega}{\partial \nu}=2\left(\left|u_{n}(x, 0)\right|+\left|v_{n}(x, 0)\right|\right)^{2_{s}^{*}-1}+A \\
& \text { on } D \times\{0\} .
\end{aligned}
$$

By choosing $D$ and $A$, we find

$$
\begin{aligned}
& -\operatorname{div}\left(y^{1-2 s} \nabla\left(\omega_{n} \pm u_{n}\right)\right)=0, \quad \text { in } \mathscr{C}_{\Omega}, \\
& \omega_{n} \pm u_{n}=0, \quad \text { on } \partial_{L} \Omega, \\
& y^{1-2 s} \frac{\partial\left(\omega_{n} \pm u_{n}\right)}{\partial \nu} \geq 0 \quad \text { on } \Omega \times\{0\} .
\end{aligned}
$$

Multiplying (22) by $\left(\omega_{n} \pm u_{n}\right)^{-}$and integrating by part, we see that

$$
\left|u_{n}\right| \leq \omega_{n}
$$

Similarly, for $v_{n}$, we have

$$
\left|v_{n}\right| \leq \omega_{n}
$$

Then by (23) and (24) we have

$$
\frac{1}{2}\left(\left|u_{n}\right|+\left|v_{n}\right|\right) \leq \omega_{n}
$$

So in the following sections we can consider just the estimates of $w_{n}$ in $\mathscr{C}_{\Omega}$.

$$
\begin{aligned}
& \mathcal{S}_{s, \alpha, \beta} \text { Let } \\
& :=\inf _{\left(w_{1}, w_{2}\right) \in E_{0}^{s}\left(\mathscr{C}_{\Omega}\right)} \frac{\int_{\mathscr{C}_{\Omega}} y^{1-2 s}\left(\left|\nabla w_{1}\right|^{2}+\left|\nabla w_{2}\right|^{2}\right) d x d y}{\left(\int_{\Omega}\left|w_{1}\right|^{\alpha}\left|w_{2}\right|^{\beta} d x\right)^{2 / 2_{s}^{*}}} .
\end{aligned}
$$


Then we have (see Lemma 2.5 [23])

$$
\mathcal{S}_{s, \alpha, \beta}=\left[\left(\frac{\alpha}{\beta}\right)^{\beta / 2_{s}^{*}}+\left(\frac{\beta}{\alpha}\right)^{\alpha / 2_{s}^{*}}\right] \mathcal{S}(s, N)
$$

where $\mathcal{S}(s, N)$ is the best Sobolev constant defined by

$$
\mathcal{S}(s, N)=\inf _{z \in H_{0, L}^{1}\left(\mathscr{C}_{\Omega}\right) \backslash\{0\}} \frac{\int_{\mathscr{C}_{\Omega}} y^{1-2 s}|\nabla z(x, y)|^{2} d x d y}{\left(\int_{\Omega}|z(x, 0)|^{2_{s}^{*}} d x\right)^{2 / 2_{s}^{*}}}
$$

which is achieved if and only if $\Omega=\mathbb{R}^{N}$ by functions $w_{\varepsilon}$ are the $s$-harmonic extensions of

$$
u_{\varepsilon}(x):=\frac{\varepsilon^{(N-2 s) / 2}}{\left(\varepsilon^{2}+|x|^{2}\right)^{(N-2 s) / 2}}, \quad \varepsilon>0, x \in \mathbb{R}^{N} .
$$

Now we introduce the "problem at infinity":

$$
\begin{aligned}
& -\operatorname{div}\left(y^{1-2 s} \nabla u\right)=0, \\
& -\operatorname{div}\left(y^{1-2 s} \nabla v\right)=0 \\
& y^{1-2 s} \frac{\partial u}{\partial \nu}=\frac{2 \alpha}{\alpha+\beta} u^{\alpha-1}(x, 0) v^{\beta}(x, 0) \\
& \text { on } \mathbb{R}^{N} \times\{0\} \text {, } \\
& y^{1-2 s} \frac{\partial v}{\partial \nu}=\frac{2 \beta}{\alpha+\beta} u^{\alpha}(x, 0) v^{\beta-1}(x, 0) \\
& \text { on } \mathbb{R}^{N} \times\{0\} \text {, } \\
& U>0 \text {, } \\
& V>0
\end{aligned}
$$

in $\mathbb{R}^{N} \times\{0\}$.

Here $\alpha>1, \beta>1$, and $\alpha+\beta=2_{s}^{*}$. Set

$$
\begin{aligned}
& U=\left(\frac{2 \alpha}{\alpha+\beta}\right)^{1 /(\alpha+\beta-2)}\left(\frac{\beta}{\alpha}\right)^{\beta / 2(\alpha+\beta-2)} u, \\
& V=\left(\frac{2 \beta}{\alpha+\beta}\right)^{1 /(\alpha+\beta-2)}\left(\frac{\alpha}{\beta}\right)^{\alpha / 2(\alpha+\beta-2)} v .
\end{aligned}
$$

Then problem (30) can be rewritten as

$$
\begin{aligned}
& -\operatorname{div}\left(y^{1-2 s} \nabla U\right)=0, \\
& -\operatorname{div}\left(y^{1-2 s} \nabla V\right)=0
\end{aligned}
$$

in $\mathbb{R}_{+}^{N+1}$,

$$
y^{1-2 s} \frac{\partial U}{\partial \nu}=U^{\alpha-1}(x, 0) V^{\beta}(x, 0)
$$

on $\mathbb{R}^{N} \times\{0\}$,

$$
y^{1-2 s} \frac{\partial V}{\partial \nu}=U^{\alpha}(x, 0) V^{\beta-1}(x, 0)
$$

$$
\text { on } \mathbb{R}^{N} \times\{0\} \text {, }
$$

$$
U>0
$$$$
V>0
$$

$$
\text { in } \mathbb{R}^{N} \times\{0\} \text {. }
$$

Lemma 3. Let $(U, V)$ be a solution of (32). Then $U(x, 0)=$ $V(x, 0)$ for all $x \in \mathbb{R}^{N}$.

Proof. Let $(U, V)$ be a solution of (32). Then

$$
\begin{aligned}
& -\operatorname{div}\left(y^{1-2 s} \nabla(U-V)\right)=0, \quad \text { in } \mathbb{R}_{+}^{N+1}, \\
& y^{1-2 s} \frac{\partial(U-V)}{\partial \nu}=U^{\alpha-1}(x, 0) V^{\beta-1}(x, 0)(V-U) \\
& U>0, \quad \text { on } \mathbb{R}^{N} \times\{0\}, \\
& V>0
\end{aligned}
$$

$$
\text { in } \mathbb{R}^{N} \times\{0\} \text {. }
$$

Multiplying (33) by $(U-V)$ and integrating by part, we obtain

$$
\begin{aligned}
& \int_{\mathbb{R}_{+}^{N+1}} y^{1-2 s}|\nabla(U-V)|^{2} d x d y \\
& =-\int_{\mathbb{R}^{N}} U^{\alpha-1}(x, 0) V^{\beta-1}(x, 0)(U-V)^{2} d x \leq 0,
\end{aligned}
$$

which implies that

$$
U(x, y)-V(x, y)=\text { const. }
$$

Note that $U>0, V>0$, in $\mathbb{R}^{N}$. It follows from (30) that $U^{\alpha-1}(x, 0) V^{\beta}(x, 0)=y^{1-2 s}(\partial U / \partial \nu)=U^{\alpha}(x, 0) V^{\beta-1}(x, 0)$ in $\mathbb{R}^{N} \times\{0\}$. Hence $U(x, 0)=V(x, 0)$.

From Proposition 6 in the Appendix, we have the global compactness result on (1). Let $\left(u_{n}, v_{n}\right)(n=1,2, \ldots)$ be a solution of problem (18) with $\varepsilon=\varepsilon_{n} \rightarrow 0$ as $n \rightarrow+\infty$, satisfying $\left\|\left(u_{n}, v_{n}\right)\right\|_{E_{0}^{s}\left(\mathscr{C}_{\Omega}\right)} \leq C$ for some constant independent of $n$. Then replacing the solution if necessary with a subsequence, there exist $k$ sequences mutually diverging scaling $\sigma_{n}^{i}$ with respective concentration points $x_{n}^{i}$ such that, as $n \rightarrow+\infty$,

$$
\begin{aligned}
u_{n}= & u_{0}+a_{0}^{-1} \sum_{i=1}^{k}\left(\sigma_{n}^{i}\right)^{(N-2 s) / 2} U\left(\sigma_{n}^{i}\left(x-x_{n}^{i}\right), 0\right) \\
& +\omega_{n}^{1}, \\
v_{n}= & v_{0}+b_{0}^{-1} \sum_{i=1}^{k}\left(\sigma_{n}^{i}\right)^{(N-2 s) / 2} U\left(\sigma_{n}^{i}\left(x-x_{n}^{i}\right), 0\right)+\omega_{n}^{2},
\end{aligned}
$$


where $\left(u_{0}, v_{0}\right) \in E_{0}^{\mathcal{S}}\left(\mathscr{C}_{\Omega}\right)$ is a weak solution of problem (12), $\left\|\left(\omega_{n}^{1}, \omega_{n}^{2}\right)\right\|_{E_{0}^{s}\left(\mathscr{C}_{\Omega}\right)} \rightarrow 0$ as $n \rightarrow+\infty, U$ achieves the constant $\mathcal{S}(s, N)$, which is given in (28), and

$$
\begin{aligned}
& a_{0}=\left(\left(\frac{2 \beta}{\alpha+\beta}\right)^{2}\left(\frac{\beta}{\alpha}\right)^{\beta-2}\right)^{1 / 2(\alpha+\beta-2)}, \\
& b_{0}=\left(\left(\frac{2 \alpha}{\alpha+\beta}\right)^{2}\left(\frac{\alpha}{\beta}\right)^{\alpha-2}\right)^{1 / 2(\alpha+\beta-2)} .
\end{aligned}
$$

As in $[7,9,10]$ we shall introduce the following facts which are essentials to prove the strong convergence of $\left\{\left(u_{n}, v_{n}\right)\right\}$ in $E_{0}^{s}\left(\mathscr{C}_{\Omega}\right)$. Among all the bubbles in (28), we can choose the slowest concentration rate, denoted by $\sigma_{n}$, which concentrates in $x_{n}$ the slowest rate. That is, the corresponding $\sigma$ is the lowest order infinity among the ones appearing in the bubbles. Note that the number of the bubbles in $\left(u_{n}, v_{n}\right)$ is finite; we can always choose a constant $\bar{C}>0$, independent of $n$, such that the region,

$$
\begin{aligned}
\mathscr{A}_{n}^{1} & :=\{Z=(x, y): Z \\
& \left.\in\left(B_{(\bar{C}+5) \sigma_{n}^{-1 / 2}}\left(x_{n}, 0\right) \backslash B_{\bar{C} \sigma_{n}^{-1 / 2}}\left(x_{n}, 0\right)\right) \cap \mathscr{C}_{\Omega}\right\},
\end{aligned}
$$

does not contain any concentration point of $\left(u_{n}, v_{n}\right)$ for every $n$. We set two thinner subsets as follows:

$$
\begin{aligned}
\mathscr{A}_{n}^{2} & :=\{Z=(x, y): Z \\
& \left.\in\left(B_{(\bar{C}+4) \sigma_{n}^{-1 / 2}}\left(x_{n}, 0\right) \backslash B_{(\bar{C}+1) \sigma_{n}^{-1 / 2}}\left(x_{n}, 0\right)\right) \cap \mathscr{C}_{\Omega}\right\}, \\
\mathscr{A}_{n}^{3} & :=\{Z=(x, y): Z \\
& \left.\in\left(B_{(\bar{C}+3) \sigma_{n}^{-1 / 2}}\left(x_{n}, 0\right) \backslash B_{(\bar{C}+2) \sigma_{n}^{-1 / 2}}\left(x_{n}, 0\right)\right) \cap \mathscr{C}_{\Omega}\right\} .
\end{aligned}
$$

Then the following integral estimates hold (see Propositions 4.1 and 4.2 in [10]).

Lemma 4. Let $\left(u_{n}, v_{n}\right)_{n \geq 1}$ be solution of (18) with $\varepsilon=\varepsilon_{n} \rightarrow 0$. Then, there exists a constant $C>0$ independent of $n$, such that, for all $p \geq 1$, one has

$$
\begin{gathered}
\int_{\mathscr{A}_{n}^{2}} y^{1-2 s}\left(\left|u_{n}\right|^{p}+\left|v_{n}\right|^{p}\right) d x d y \leq C \sigma_{n}^{-\left(N+2_{s}^{*}\right) / 2_{s}^{*}}, \\
\int_{\mathscr{A}_{n}^{2} \cap\{y=0\}}\left(\left|u_{n}\right|^{p}+\left|v_{n}\right|^{p}\right) d x \leq C \sigma_{n}^{-N / 2} .
\end{gathered}
$$

Moreover, one has

$$
\int_{\mathscr{A}_{n}^{3}} y^{1-2 s}\left(\left|\nabla u_{n}\right|^{2}+\left|\nabla v_{n}\right|^{2}\right) d x d y \leq C \sigma_{n}^{-N / 2_{s}^{*}} .
$$

Proof. For the proof of this lemma, we use inequality (25) and we refer to [10, Propositions 4.1, 4.2].

Note that $X=(x, y) \in \mathbb{R}_{+}^{N+1}$, and we have the following local Pohozaev identity.

Lemma 5. Let $\alpha_{n}=\alpha-\varepsilon_{n}, \beta_{n}=\beta-\varepsilon_{n}$, and $\varepsilon=\varepsilon_{n} \rightarrow 0$ as $n \rightarrow+\infty$ and $B_{n}$ be any bounded set in $\mathscr{C}_{\Omega}$. Then one has the local Pohozaev identity on $B_{n} \subset \mathscr{C}_{\Omega}$ associated for equations of (18):

$$
\begin{aligned}
& \frac{\varepsilon_{n}(N-2 s)^{2}}{N-\varepsilon_{n}(N-2 s)} \int_{B_{n} \cap\{y=0\}}\left|u_{n}\right|^{\alpha_{n}}\left|v_{n}\right|^{\beta_{n}} d x \\
& +s \int_{B_{n} \cap\{y=0\}}\left(\lambda u_{n}^{2}+\mu v_{n}^{2}\right) d x \\
& =\int_{\partial\left(B_{n} \cap\{y=0\}\right)} \frac{2}{\alpha_{n}+\beta_{n}}\left|u_{n}\right|^{\alpha_{n}}\left|v_{n}\right|^{\beta_{n}}\left(\left(x-x_{0}\right)\right. \\
& \cdot \nu) d \sigma+\frac{1}{2} \int_{\partial\left(B_{n} \cap\{y=0\}\right)}\left(\lambda u_{n}^{2}+\mu v_{n}^{2}\right)\left(\left(x-x_{0}\right)\right. \\
& \cdot v) d \sigma+\frac{N-2 s}{2} \int_{\partial B_{n} \cap\{y>0\}} y^{1-2 s}\left(u_{n} \frac{\partial u_{n}}{\partial \nu}\right. \\
& \left.+v_{n} \frac{\partial v_{n}}{\partial v}\right) d \sigma-\frac{1}{2} \\
& \cdot \int_{\partial B_{n} \cap\{y>0\}} y^{1-2 s}\left(\left|\nabla u_{n}\right|^{2}+\left|\nabla v_{n}\right|^{2}\right)\left(\left(X-z_{0}\right)\right. \\
& \cdot v) d \sigma \\
& +\int_{\partial B_{n} \cap\{y>0\}} y^{1-2 s}\left(\left(\nabla u_{n} \cdot\left(X-z_{0}\right)\right) \frac{\partial u_{n}}{\partial \nu}\right. \\
& \left.+\left(\nabla v_{n} \cdot\left(X-z_{0}\right)\right) \frac{\partial v_{n}}{\partial v}\right) d \sigma
\end{aligned}
$$

where $v$ is the outward unit normal to $\partial B_{n}$ and $z_{0}=\left(x_{0}, 0\right)$, with $x_{0}$ being a point in $\mathbb{R}^{N}$.

Proof. Using the divergence theorem, we get

$$
\begin{aligned}
\frac{N-2 s}{2} \int_{\partial B_{n}} y^{1-2 s}\left(u_{n} \frac{\partial u_{n}}{\partial \nu}+v_{n} \frac{\partial v_{n}}{\partial \nu}\right) d \sigma= & \frac{1}{2} \int_{\partial B_{n}} y^{1-2 s}\left(\left|\nabla u_{n}\right|^{2}+\left|\nabla v_{n}\right|^{2}\right)\left(\left(X-z_{0}\right) \cdot v\right) d \sigma \\
& -\int_{\partial B_{n}} y^{1-2 s}\left(\left(\nabla u_{n} \cdot\left(X-z_{0}\right)\right) \frac{\partial u_{n}}{\partial \nu}+\left(\nabla v_{n} \cdot\left(X-z_{0}\right)\right) \frac{\partial v_{n}}{\partial \nu}\right) d \sigma,
\end{aligned}
$$


where $v$ is the outward unit normal to $\partial B_{n}$ and $z_{0}=\left(x_{0}, 0\right)$, with $x_{0}$ being a point in $\mathbb{R}^{N}$.

Since $\left(u_{n}, v_{n}\right)$ is solution of (18), we have the following equations:

$$
y^{1-2 s} u_{n} \frac{\partial u_{n}}{\partial v}=\frac{2 \alpha_{n}}{\alpha_{n}+\beta_{n}}\left|u_{n}\right|^{\alpha_{n}}\left|v_{n}\right|^{\beta_{n}}+\lambda u_{n}^{2}
$$$$
\text { on } y=0 \text {, }
$$

$$
y^{1-2 s} v_{n} \frac{\partial v_{n}}{\partial v}=\frac{2 \beta_{n}}{\alpha_{n}+\beta_{n}}\left|u_{n}\right|^{\alpha_{n}}\left|v_{n}\right|^{\beta_{n}}+\mu v_{n}^{2}
$$

on $y=0$.

$$
\begin{aligned}
& \frac{N-2 s}{2} \int_{B_{n} \cap\{y=0\}}\left(2\left|u_{n}\right|^{\alpha_{n}}\left|v_{n}\right|^{\beta_{n}}+\lambda u_{n}^{2}+\mu v_{n}^{2}\right) d x \\
& \quad+\frac{N-2 s}{2} \int_{\partial B_{n} \cap\{y>0\}} y^{1-2 s}\left(u_{n} \frac{\partial u_{n}}{\partial \nu}+v_{n} \frac{\partial v_{n}}{\partial \nu}\right) d \sigma=\frac{1}{2} \int_{B_{n} \cap\{y=0\}} y^{1-2 s}\left(\left|\nabla u_{n}\right|^{2}+\left|\nabla v_{n}\right|^{2}\right)\left(\left(x-x_{0}\right) \cdot v\right) d x \\
& +\frac{1}{2} \int_{\partial B_{n} \cap\{y>0\}} y^{1-2 s}\left(\left|\nabla u_{n}\right|^{2}+\left|\nabla v_{n}\right|^{2}\right)(X \cdot \nu) d \sigma-\int_{B_{n} \cap\{y=0\}} y^{1-2 s}\left(\left(\nabla u_{n} \cdot\left(x-x_{0}\right)\right) \frac{\partial u_{n}}{\partial \nu}+\left(\nabla v_{n} \cdot\left(x-x_{0}\right)\right) \frac{\partial v_{n}}{\partial \nu}\right) d x \\
& -\int_{\partial B_{n} \cap\{y>0\}} y^{1-2 s}\left(\left(\nabla u_{n} \cdot\left(X-z_{0}\right)\right) \frac{\partial u_{n}}{\partial \nu}+\left(\nabla v_{n} \cdot\left(X-z_{0}\right)\right) \frac{\partial v_{n}}{\partial \nu}\right) d \sigma .
\end{aligned}
$$

We obtain, from (43) and (44),

Noting that the dot product $\left(\left(x-x_{0}\right) \cdot v\right)=0$, on $B_{n} \cap\{y=0\}$, we obtain

$$
\begin{aligned}
& \frac{1}{2} \int_{B_{n} \cap\{y=0\}} y^{1-2 s}\left(\left|\nabla u_{n}\right|^{2}+\left|\nabla v_{n}\right|^{2}\right)\left(\left(x-x_{0}\right) \cdot v\right) d x \\
& \quad=0 .
\end{aligned}
$$

Moreover, we have

$$
\begin{aligned}
& \int_{B_{n} \cap\{y=0\}} y^{1-2 s}\left(\left(\nabla u_{n} \cdot\left(x-x_{0}\right)\right) \frac{\partial u_{n}}{\partial \nu}+\left(\nabla v_{n} \cdot\left(x-x_{0}\right)\right) \frac{\partial v_{n}}{\partial \nu}\right) d x=\int_{B_{n} \cap\{y=0\}}\left(\nabla u_{n} \cdot\left(x-x_{0}\right)\right) \\
& \cdot\left(\frac{2 \alpha_{n}}{\alpha_{n}+\beta_{n}}\left|u_{n}\right|^{\alpha_{n}}\left|v_{n}\right|^{\beta_{n}}+\lambda u_{n}^{2}\right) d x+\int_{B_{n} \cap\{y=0\}}\left(\nabla v_{n} \cdot\left(x-x_{0}\right)\right)\left(\frac{2 \beta_{n}}{\alpha_{n}+\beta_{n}}\left|u_{n}\right|^{\alpha_{n}}\left|v_{n}\right|^{\beta_{n}}+\mu v_{n}^{2}\right) d x \\
& =\int_{B_{n} \cap\{y=0\}} \nabla\left(\frac{2}{\alpha_{n}+\beta_{n}}\left|u_{n}\right|^{\alpha_{n}}\left|v_{n}\right|^{\beta_{n}}+\frac{1}{2}\left(\lambda u_{n}^{2}+\mu v_{n}^{2}\right)\right) \cdot\left(x-x_{0}\right) d x \\
& =-N \int_{B_{n} \cap\{y=0\}}\left(\frac{2}{\alpha_{n}+\beta_{n}}\left|u_{n}\right|^{\alpha_{n}}\left|v_{n}\right|^{\beta_{n}}+\frac{1}{2}\left(\lambda u_{n}^{2}+\mu v_{n}^{2}\right)\right) d x \\
& \quad+\int_{\partial\left(B_{n} \cap\{y=0\}\right)}\left(\frac{2}{\alpha_{n}+\beta_{n}}\left|u_{n}\right|^{\alpha_{n}}\left|v_{n}\right|^{\beta_{n}}+\frac{1}{2}\left(\lambda u_{n}^{2}+\mu v_{n}^{2}\right)\right)\left(\left(x-x_{0}\right) \cdot v\right) d \sigma .
\end{aligned}
$$

Therefore, from (45), (46), and (47), we infer that (44) holds.

Proof of Proposition 2. Let $\left(u_{n}, v_{n}\right)_{n \geq 1}$ be a bounded sequence in $E_{0}^{s}\left(\mathscr{C}_{\Omega}\right)$ composed of solutions for (18). Thus, in order to prove the $E_{0}^{s}\left(\mathscr{C}_{\Omega}\right)$-strong convergence in (36), we just need to show that the bubbles $\left(\sigma_{n}^{i}\right)^{(N-2 s) / 2} U\left(\sigma_{n}^{i}\left(x-x_{n}^{i}\right), 0\right)(1 \leq i \leq$ $k$ ) in (36) will not appear in the decomposition of $u_{n}$ and $v_{n}$. Since the proof is similar to that of Lemma 6.1 in [9], here 
we only give a sketch of it. From Lemma 5, for the solution concentrating sequence $\left(u_{n}, v_{n}\right)$ of (18) with $\varepsilon=\varepsilon_{n} \rightarrow 0$, we have the local Pohozaev identity on

$$
\begin{aligned}
& B_{n}:=B_{t_{n} \sigma_{n}^{-1 / 2}}\left(\left(x_{n}, 0\right)\right) \cap \mathscr{C}_{\Omega} \subset \mathbb{R}^{N+1} \\
& \frac{4 \varepsilon_{n} N}{2_{s}^{*}\left(2_{s}^{*}-2 \varepsilon_{n}\right)} \int_{B_{n} \cap\{y=0\}}\left|u_{n}\right|^{\alpha_{n}}\left|v_{n}\right|^{\beta_{n}} d x \\
& \quad+s \int_{B_{n} \cap\{y=0\}}\left(\lambda u_{n}^{2}+\mu v_{n}^{2}\right) d x=\frac{2}{\alpha_{n}+\beta_{n}} \\
& \quad \cdot \int_{\partial\left(B_{n} \cap\{y=0\}\right)}\left|u_{n}\right|^{\alpha_{n}}\left|v_{n}\right|^{\beta_{n}}\left(\left(X-z_{0}\right) \cdot \nu\right) d \sigma+\frac{1}{2} \\
& \quad \cdot \int_{\partial\left(B_{n} \cap\{y=0\}\right)}\left(\lambda u_{n}^{2}+\mu v_{n}^{2}\right)\left(\left(X-z_{0}\right) \cdot \nu\right) d \sigma \\
& \quad+\frac{N-2 s}{2} \int_{\partial B_{n} \cap\{y>0\}} y^{1-2 s}\left(u_{n} \frac{\partial u_{n}}{\partial \nu}+v_{n} \frac{\partial v_{n}}{\partial \nu}\right) d \sigma \\
& \quad-\frac{1}{2} \int_{\partial B_{n} \cap\{y>0\}} y^{1-2 s}\left(\left|\nabla u_{n}\right|^{2}+\left|\nabla v_{n}\right|^{2}\right)\left(\left(X-z_{0}\right)\right. \\
&\quad \cdot \nu) d \sigma \\
& \quad+\int_{\partial B_{n} \cap\{y>0\}} y^{1-2 s}\left(\left(\nabla u_{n} \cdot X-z_{0}\right) \frac{\partial u_{n}}{\partial \nu}\right. \\
&\left.+\left(\nabla v_{n} \cdot X-z_{0}\right) \frac{\partial v_{n}}{\partial \nu}\right) d \sigma,
\end{aligned}
$$

where $v$ is the outward unit normal to $\partial B_{n}$ and $z_{0}=\left(x_{0}, 0\right)$, $x_{0} \in \mathbb{R}^{N}$. We decompose

$$
\partial B_{n} \cap\{y>0\}=\partial_{i} B_{n} \cup \partial_{e} B_{n}
$$

where $\partial_{i} B_{n}:=\partial B_{n} \cap \mathscr{C}_{\Omega}$ and $\partial_{e} B_{n}:=B_{n} \cap \partial_{L} \mathscr{C}_{\Omega}$.

We consider two different cases:

(i) $B_{t_{n} \sigma_{n}^{-1 / 2}}\left(\left(x_{n}, 0\right)\right) \cap\{0\} \cap\left(\mathbb{R}^{N+1} \backslash \mathscr{C}_{\Omega}\right) \neq \emptyset$.

(ii) $B_{t_{n} \sigma_{n}^{-1 / 2}}\left(\left(x_{n}, 0\right)\right) \cap\{0\} \subset \mathscr{C}_{\Omega}$.

In case (i), we take $x_{0} \in \mathbb{R}^{N} \backslash \Omega$ with $\left|x_{0}-x_{n}\right| \leq 2 t_{n} \sigma_{n}^{-1 / 2}$ and $v \cdot\left(X-\left(x_{0}, 0\right)\right) \leq 0$ in $\partial_{e} B_{n}$, where $v$ is the outward unit normal to $\partial_{L} \mathscr{C}_{\Omega}$.

Since $\left(u_{n}, v_{n}\right)=(0,0)$ on $\partial_{L} \mathscr{C}_{\Omega}$, we find

$$
\begin{aligned}
& -\frac{1}{2} \int_{\partial B_{n} \cap\{y>0\}} y^{1-2 s}\left(\left|\nabla u_{n}\right|^{2}+\left|\nabla v_{n}\right|^{2}\right)\left(\left(X-z_{0}\right) \cdot v\right) d \sigma \\
& +\int_{\partial B_{n} \cap\{y>0\}} y^{1-2 s}\left(\left(\nabla u_{n} \cdot X-z_{0}\right) \frac{\partial u_{n}}{\partial \nu}\right. \\
& \left.+\left(\nabla v_{n} \cdot X-z_{0}\right) \frac{\partial v_{n}}{\partial \nu}\right) d \sigma=\frac{1}{2} \\
& +\int_{\partial B_{n} \cap\{y>0\}} y^{1-2 s}\left(\left|\nabla u_{n}\right|^{2}+\left|\nabla v_{n}\right|^{2}\right)\left(\left(X-z_{0}\right)\right. \\
& \cdot v) d \sigma \leq 0 .
\end{aligned}
$$

In case (ii), $\partial_{e} B_{n}=\emptyset$; we take a point $x_{0}=x_{n}$.
Since $\left(u_{n}, v_{n}\right)=(0,0)$ on $\partial_{L} \mathscr{C}_{\Omega}$, and, for $n$ large enough $4 \varepsilon_{n} N / 2_{s}^{*}\left(2_{s}^{*}-2 \varepsilon_{n}\right)>0$, we obtain

$$
\begin{aligned}
& s \int_{B_{n} \cap\{y=0\}}\left(\lambda u_{n}^{2}+\mu v_{n}^{2}\right) d x \leq \int_{\partial_{i}\left(B_{n} \cap\{0\}\right)} \frac{2}{\alpha_{n}+\beta_{n}}\left|u_{n}\right|^{\alpha_{n}} \\
& \cdot\left|v_{n}\right|^{\beta_{n}}\left(\left(X-z_{0}\right) \cdot \nu\right) d \sigma+\frac{1}{2} \\
& \cdot \int_{\left(\partial_{i} B_{n}\right) \cap\{y=0\}}\left(\lambda u_{n}^{2}+\mu v_{n}^{2}\right)\left(\left(X-z_{0}\right) \cdot \nu\right) d \sigma \\
& \quad+\frac{N-2 s}{2} \int_{\partial_{i} B_{n}} y^{1-2 s}\left(u_{n} \frac{\partial u_{n}}{\partial \nu}+v_{n} \frac{\partial v_{n}}{\partial \nu}\right) d \sigma-\frac{1}{2} \\
& \quad \cdot \int_{\partial_{i} B_{n}} y^{1-2 s}\left(\left|\nabla u_{n}\right|^{2}+\left|\nabla v_{n}\right|^{2}\right)\left(\left(X-z_{0}\right) \cdot \nu\right) d \sigma \\
& \quad+\int_{\partial_{i} B_{n}} y^{1-2 s}\left(\left(\nabla u_{n} \cdot X-z_{0}\right) \frac{\partial u_{n}}{\partial \nu}\right. \\
& \left.\quad+\left(\nabla v_{n} \cdot X-z_{0}\right) \frac{\partial v_{n}}{\partial \nu}\right) d \sigma .
\end{aligned}
$$

By assumption, $\lambda, \mu \geq 0$ and $\lambda+\mu>0$; we may assume that $\lambda>0$. Set $B_{n}^{\prime}=B_{\sigma_{n}^{-1}}\left(x_{n}, 0\right) \cap \mathscr{C}_{\Omega}$. Recalling that, by (36), we have the decomposition $u_{n}=u_{n}^{0}+u_{n}^{1}+u_{n}^{2}$, where $u_{n}^{1}=u_{0}$, $u_{n}^{2}=a_{0}^{-1} \sum_{j=1}^{k}\left(\sigma_{n}^{i}\right)^{(N-2 s) / 2} U\left(\sigma_{n}^{i}\left(x-x_{n}^{i}\right), 0\right)$, and $u_{n}^{0}=u_{n}-u_{n}^{1}-u_{n}^{2}$. $\left\|u_{n}^{0}\right\|_{E_{0}^{s}\left(\mathscr{C}_{\Omega}\right)} \rightarrow 0$ as $n \rightarrow \infty$. Then we deduce that, for $n$ large enough, $B_{n}^{\prime} \subset B_{n}$

$$
\begin{aligned}
\int_{B_{n} \cap\{y=0\}} u_{n}^{2} d x \geq & \int_{B_{n}^{\prime} \cap\{y=0\}} u_{n}^{2} d x \\
\geq & \frac{1}{2} \int_{B_{n}^{\prime} \cap\{y=0\}}\left|u_{n}^{2}\right|^{2} d x \\
& -2 \int_{B_{n}^{\prime} \cap\{y=0\}}\left(\left|u_{n}^{1}\right|^{2}+\left|u_{n}^{0}\right|^{2}\right) d x .
\end{aligned}
$$

After a direct calculation, we have

$$
\begin{aligned}
& \int_{B_{n}^{\prime} \cap\{y=0\}}\left|u_{n}^{2}\right|^{2} d x \geq C \sigma_{n}^{-2 s}, \\
& \int_{B_{n}^{\prime} \cap\{y=0\}}\left|u_{n}^{1}\right|^{2} d x \leq C \sigma_{n}^{-N}, \\
& \int_{B_{n}^{\prime} \cap\{y=0\}}\left|u_{n}^{0}\right|^{2} d x \leq C\left\|u_{n}^{0}\right\|_{L^{2 *}(\Omega)}^{2} \sigma_{n}^{-2 s} .
\end{aligned}
$$

Note that $\left\|u_{n}^{0}\right\|_{E_{0}^{s}\left(\mathscr{C}_{\Omega}\right)} \rightarrow 0$, as $n \rightarrow \infty$. Inserting (54) into (53), we get for $n$ large enough

$$
\int_{B_{n} \cap\{y=0\}} \lambda u_{n}^{2} d x \geq C \sigma_{n}^{-2 s} .
$$


By the choice of $z_{0}=\left(x_{0}, 0\right)$, as in [10], we only need to consider the right-hand side of (52) on $\partial_{i} B_{n}$. By applying Hölder inequality and using Lemma 4, we get

$$
\begin{aligned}
& \frac{2}{\alpha_{n}+\beta_{n}} \int_{\partial_{i}\left(B_{n} \cap\{y=0\}\right)}\left|u_{n}\right|^{\alpha_{n}}\left|v_{n}\right|^{\beta_{n}}\left(\left(X-z_{0}\right) \cdot \nu\right) d \sigma+\frac{1}{2} \\
& \cdot \int_{\left(\partial_{i} B_{n}\right) \cap\{y=0\}}\left(\lambda u_{n}^{2}+\mu v_{n}^{2}\right)\left(\left(X-z_{0}\right) \cdot \nu\right) d \sigma \\
& +\frac{N-2 s}{2} \int_{\partial_{i} B_{n}} y^{1-2 s}\left(u_{n} \frac{\partial u_{n}}{\partial \nu}+v_{n} \frac{\partial v_{n}}{\partial \nu}\right) d \sigma-\frac{1}{2} \\
& \cdot \int_{\partial_{i} B_{n}} y^{1-2 s}\left(\left|\nabla u_{n}\right|^{2}+\left|\nabla v_{n}\right|^{2}\right)\left(\left(X-z_{0}\right) \cdot \nu\right) d \sigma \\
& +\int_{\partial_{i} B_{n}} y^{1-2 s}\left(\left(\nabla u_{n} \cdot X-z_{0}\right) \frac{\partial u_{n}}{\partial \nu}\right. \\
& \left.+\left(\nabla v_{n} \cdot X-z_{0}\right) \frac{\partial v_{n}}{\partial \nu}\right) d \sigma \\
& \leq C \sigma_{n}^{-1 / 2}\left(\int_{\partial_{i}\left(B_{n} \cap\{y=0\}\right)}\left|u_{n}\right|^{\alpha_{n}+\beta_{n}} d \sigma\right)^{\alpha_{n} /\left(\alpha_{n}+\beta_{n}\right)} \\
& \cdot\left(\int_{\partial_{i}\left(B_{n} \cap\{y=0\}\right)}\left|v_{n}\right|^{\alpha_{n}+\beta_{n}} d \sigma\right)^{\beta_{n} /\left(\alpha_{n}+\beta_{n}\right)} \\
& +\int_{\partial_{i}\left(B_{n} \cap\{y=0\}\right)}\left(\left|\nabla u_{n}\right|^{2}+\left|\nabla v_{n}\right|^{2}\right)\left|x-x_{0}\right| d \sigma \\
& +\left(\int_{\partial_{i}\left(B_{n} \cap\{y=0\}\right)}\left(\left|\nabla u_{n}\right|^{2}+\left|\nabla v_{n}\right|^{2}\right) d \sigma\right)^{1 / 2} \\
& \cdot\left(\int_{\partial_{i}\left(B_{n} \cap\{y=0\}\right)}\left(\left|u_{n}\right|^{2}+\left|v_{n}\right|^{2}\right) d \sigma\right)^{1 / 2} \\
& \leq C \sigma_{n}^{-(N-2 s) / 2} \text {. }
\end{aligned}
$$

Inserting (56) and (57) into (52), we obtain

$$
\sigma_{n}^{-2 s} \leq C \sigma_{n}^{-(N-2 s) / 2}
$$

which is a contradiction for $n$ large enough due to $N>6 s$.

\section{Proof of Theorem 1}

For any positive integer $k$, define the $Z_{2}$-homotopy class $\mathscr{F}_{k}$ as follows:

$$
\begin{aligned}
\mathscr{F}_{k} & :=\{A: A \\
& \left.\subset E_{0}^{s}\left(\mathscr{C}_{\Omega}\right) \text { is compact, } Z_{2} \text {-invariant, } \gamma(A) \geq k\right\},
\end{aligned}
$$

where the genus $\gamma(A)$ is the smallest integer $m$, such that there exists an odd map $\phi \in \mathscr{C}\left(A, \mathbb{R}^{m} \backslash\{0\}\right)$. For $k=1,2, \ldots$, we define the min-max value as (e.g., see p. 134 in [26])

$$
c_{k, \varepsilon}=\min _{A \in \mathscr{F}_{k}(u, v) \in A} \max _{\lambda, \mu} I^{\varepsilon}(u, v) .
$$

It follows from Corollary 7.12 in [26] that, for each small $\varepsilon>0, c_{k, \varepsilon}$ is critical value of $I_{\lambda, \mu}^{\varepsilon}$, since $I_{\lambda, \mu}^{\varepsilon}$ satisfies the PalaisSmale condition. Thus problem (18) has a solution $\left(u_{k, \varepsilon}, v_{k, \varepsilon}\right)$ such that $I_{\lambda, \mu}^{\varepsilon}\left(u_{k, \varepsilon}, v_{k, \varepsilon}\right)=c_{k, \varepsilon}$. Note that $I_{\lambda, \mu}^{\varepsilon}\left(t u_{k, \varepsilon}, t v_{k, \varepsilon}\right) \rightarrow$ $-\infty$ uniformly with respect to $\varepsilon>0$ as $t \rightarrow+\infty$; hence, $c_{k, \varepsilon}$ is uniformly bounded with respect to $\varepsilon$ for each fixed $k$. By a direct calculation, we find $\left\|\left(u_{k, \varepsilon}, v_{k, \varepsilon}\right)\right\|_{E_{0}^{s}\left(\mathscr{C}_{\Omega}\right)} \leq C$ uniformly with respect to $\varepsilon$ for each fixed $k$. So now, we can apply Proposition 2 and obtain a subsequence of $\left(u_{k, \varepsilon_{n}}, v_{k, \varepsilon_{n}}\right)_{n \geq 1}$, such that, as $n \rightarrow+\infty,\left(u_{k, \varepsilon_{n}}, v_{k, \varepsilon_{n}}\right) \rightarrow\left(u_{k}, v_{k}\right)$ strongly in $E_{0}^{\mathcal{S}}\left(\mathscr{C}_{\Omega}\right)$ for some $\left(u_{k}, v_{k}\right)$ and $c_{k, \varepsilon_{n}} \rightarrow c_{k}$. Then $\left(u_{k}, v_{k}\right)$ is solution of (1) and $I_{\lambda, \mu}\left(u_{k}, v_{k}\right)=c_{k}$. We are now ready to show that $I_{\lambda, \mu}$ has infinitely many critical point solutions. Note that $c_{k}$ is nondecreasing in $k$. By an argument similar to the one used in the proof of Theorem 1.1 in [9] we distinguish two cases.

Case 1. Suppose that there are $1<k_{1}<\cdots k_{i}<\cdots$, satisfying

$$
c_{k_{1}}<\cdots<c_{k_{i}}<\cdots .
$$

In this case, we have infinitely many distinct critical points and, therefore, infinitely many solutions.

Case 2. We assume, in this case, that for some positive integer $m, c_{k}=c$ for all $k \geq m$. Suppose that, for any $\delta>0, I_{\lambda, \mu}$ has a critical point $(u, v)$ with $I_{\lambda, \mu}(u, v) \in(c-\delta, c+\delta)$ and $I_{\lambda, \mu}(u, v) \neq c$. In this case, we are done. So from now on we assume that there exists $\delta>0$ such that $I_{\lambda, \mu}$ has no critical point $(u, v)$ with $I_{\lambda, \mu}(u, v) \in(c-\delta, c) \cup(c, c+\delta)$. In this case, using the deformation argument, we can prove that

$$
\gamma\left(K_{c}\right) \geq 2 \text {, }
$$

where $K_{c}=\left\{(u, v) \in X: I_{\lambda, \mu}(u, v)=c, I_{\lambda, \mu}^{\prime}(u, v)=0\right\}$. As a consequence, $I_{\lambda, \mu}$ has infinitely many critical points. Thus we can obtain infinitely many solutions for problem (1).

\section{Appendix}

In this section, we give a global compactness result in the following proposition.

Proposition 6. Suppose that $\left(u_{n}, v_{n}\right)_{n \geq 1}$ is a solution of (18) with $\varepsilon=\varepsilon_{n} \rightarrow 0$, satisfying $\left\|\left(u_{n}, v_{n}\right)\right\|_{E_{0}^{s}\left(\mathscr{C}_{\Omega}\right)} \leq C$ for that $C$ is the constant independent of $n$. Then

(i) $\left(u_{n}, v_{n}\right)$ can be decomposed as

$$
\begin{aligned}
u_{n}= & u_{0}+a_{0}^{-1} \sum_{i=1}^{k}\left(\sigma_{n}^{i}\right)^{(N-2 s) / 2} U\left(\sigma_{n}^{i}\left(x-x_{n}^{i}\right), 0\right) \\
& +\omega_{n}^{1}, \\
v_{n}= & v_{0}+b_{0}^{-1} \sum_{i=1}^{k}\left(\sigma_{n}^{i}\right)^{(N-2 s) / 2} U\left(\sigma_{n}^{i}\left(x-x_{n}^{i}\right), 0\right) \\
& +\omega_{n}^{2},
\end{aligned}
$$

where $\left(u_{0}, v_{0}\right) \in E_{0}^{s}\left(\mathscr{C}_{\Omega}\right)$ is a weak solution of problem (12),

$\left\|\left(\omega_{n}^{1}, \omega_{n}^{2}\right)\right\|_{E_{0}^{s}\left(\mathscr{C}_{\Omega}\right)} \rightarrow 0$, as $n \rightarrow+\infty, U$ achieves the constant $\mathcal{S}(s, N)$, which is given in (28); and

$$
\begin{aligned}
& a_{0}=\left(\left(\frac{2 \beta}{\alpha+\beta}\right)^{2}\left(\frac{\beta}{\alpha}\right)^{\beta-2}\right)^{1 / 2(\alpha+\beta-2)}, \\
& b_{0}=\left(\left(\frac{2 \alpha}{\alpha+\beta}\right)^{2}\left(\frac{\alpha}{\beta}\right)^{\alpha-2}\right)^{1 / 2(\alpha+\beta-2)},
\end{aligned}
$$


for $i=1, \ldots, k, x_{n, i} \in \Omega$ with $\sigma_{n}^{i} d\left(x_{n, i}, \partial \Omega\right) \rightarrow+\infty$, $\sigma_{n}^{i}\left|x_{n, i}\right| \rightarrow+\infty$

(ii) for $i, j=1, \ldots, k$, if $i \neq j$, then, as $n \rightarrow+\infty$,

$$
\frac{\sigma_{n}^{j}}{\sigma_{n}^{i}}+\frac{\sigma_{n}^{i}}{\sigma_{n}^{j}}+\sigma_{n}^{i} \sigma_{n}^{j}\left|x_{n, i}-x_{n, j}\right|^{2} \longrightarrow+\infty .
$$

Proof. The proof follows without difficulty by modifying the proof of the concentration compactness result for (2) (see [10, 15]) and using Lemma 3. We omit the details for the sake of simplicity.

\section{Competing Interests}

The authors declare that they have no competing interests.

\section{References}

[1] L. Caffarelli, "Non-local diffusions, drifts and games," in Nonlinear Partial Differential Equations, vol. 7, pp. 37-52, Springer, 2012.

[2] E. Valdinoci, "From the long jump random walk to the fractional laplacian," 2009, https://arxiv.org/abs/0901.3261.

[3] J. L. Vázquez, "Recent progress in the theory of nonlinear diffusion with fractional Laplacian operators," Discrete \& Continuous Dynamical Systems, vol. 7, no. 4, pp. 857-885, 2014.

[4] H. Brézis and L. Nirenberg, "Positive solutions of nonlinear elliptic equations involving critical sobolev exponents," Communications on Pure and Applied Mathematics, vol. 36, no. 4, pp. 437-477, 1983.

[5] P.-L. Lions, "The concentration-compactness principle in the calculus of variations. The limit case, I," Revista Matemática Iberoamericana, vol. 1, no. 1, pp. 145-201, 1985.

[6] M. Struwe, "A global compactness result for elliptic boundary value problems involving limiting nonlinearities," Mathematische Zeitschrift, vol. 187, no. 4, pp. 511-517, 1984.

[7] G. Devillanova and S. Solimini, "Concentration estimates and multiple solutions to elliptic problems at critical growth," Advances in Differential Equations, vol. 7, no. 10, pp. 1257-1280, 2002.

[8] A. Bahri and P.-L. Lions, "Morse index of some min-max critical points. I. Application to multiplicity results," Communications on Pure and Applied Mathematics, vol. 41, no. 8, pp. 1027-1037, 1988.

[9] D. Cao, S. Peng, and S. Yan, "Infinitely many solutions for $p$ Laplacian equation involving critical Sobolev growth," Journal of Functional Analysis, vol. 262, no. 6, pp. 2861-2902, 2012.

[10] S. Yan, J. Yang, and X. Yu, "Equations involving fractional laplacian operator: compactness and application," Journal of Functional Analysis, vol. 269, no. 1, pp. 47-79, 2015.

[11] C. O. Alves, D. C. De Morais Filho, and M. A. S. Souto, "On systems of elliptic equations involving subcritical or critical Sobolev exponents," Nonlinear Analysis: Theory, Methods and Applications, vol. 42, no. 5, pp. 771-787, 2000.

[12] X. Cheng and S. Ma, "Existence of three nontrivial solutions for elliptic systems with critical exponents and weights," Nonlinear Analysis: Theory, Methods and Applications, vol. 69, no. 10, pp. 3537-3548, 2008.
[13] Z. Liu and P. Han, "Infinitely many solutions for elliptic systems with critical exponents," Journal of Mathematical Analysis and Applications, vol. 353, no. 2, pp. 544-552, 2009.

[14] M. Willem, Minimax Theorems, Progress in Nonlinear Differential Equations and Their Applications, Birkhäuser, Boston, Mass, USA, 1996.

[15] L. Caffarelli and L. Silvestre, "An extension problem related to the fractional laplacian," Communications in Partial Differential Equations, vol. 32, no. 8, pp. 1245-1260, 2007.

[16] B. Barrios, E. Colorado, A. de Pablo, and U. Sánchez, “On some critical problems for the fractional Laplacian operator," Journal of Differential Equations, vol. 252, no. 11, pp. 6133-6162, 2012.

[17] A. Fiscella, G. Molica Bisci, and R. Servadei, "Bifurcation and multiplicity results for critical nonlocal fractional Laplacian problems," Bulletin des Sciences Mathématiques, vol. 140, no. 1, pp. 14-35, 2016.

[18] S. Secchi, "Perturbation results for some nonlinear equations involving fractional operators," Differential Equations \& Applications, vol. 5, no. 2, pp. 221-236, 2013.

[19] R. Servadei and E. Valdinoci, "Fractional Laplacian equations with critical Sobolev exponent," Revista Matemática Complutense, vol. 28, no. 3, pp. 655-676, 2015.

[20] X. Shang, J. Zhang, and Y. Yang, "Positive solutions of nonhomogeneous fractional laplacian problem with critical exponent," Communications on Pure and Applied Analysis, vol. 13, no. 2, pp. 567-584, 2014.

[21] Z. Shen and F. Gao, "Existence of solutions for a fractional laplacian equation with critical nonlinearity," Abstract and Applied Analysis, vol. 2013, Article ID 638425, 9 pages, 2013.

[22] Z. Shen and F. Gao, "On the existence of solutions for the critical fractional laplacian equation in $\mathbb{R}^{N}$, Abstract and Applied Analysis, vol. 2014, Article ID 143741, 10 pages, 2014.

[23] X. He, M. Squassina, and W. Zou, "The Nehari manifold for fractional systems involving critical nonlinearities," http://arxiv.org/abs/1509.02713.

[24] A. Ambrosetti and P. H. Rabinowitz, "Dual variational methods in critical point theory and applications," Journal of Functional Analysis, vol. 14, no. 4, pp. 349-381, 1973.

[25] N. Ghoussoub and C. Yuan, "Multiple solutions for quasi-linear PDEs involving the critical Sobolev and Hardy exponents," Transactions of the American Mathematical Society, vol. 352, no. 12, pp. 5703-5743, 2000.

[26] N. Ghoussoub, Duality and Perturbation Methods in Critical Point Theory, vol. 107 of Cambridge Tracts in Mathematics, Cambridge University Press, 1993. 


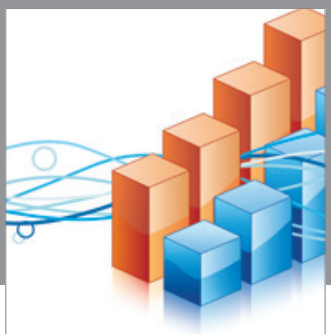

Advances in

Operations Research

vatem alat4

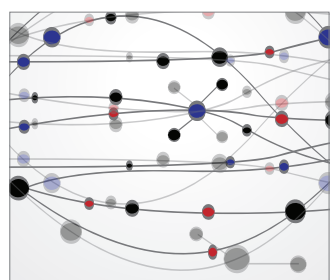

\section{The Scientific} World Journal
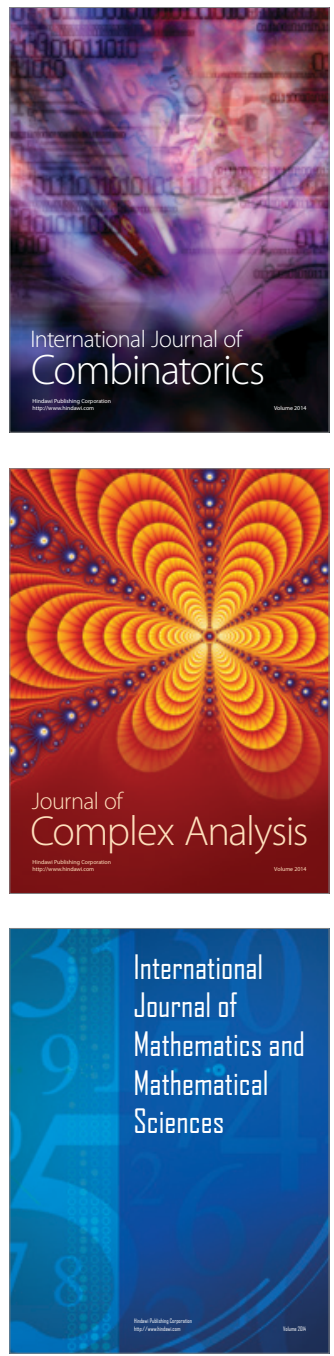
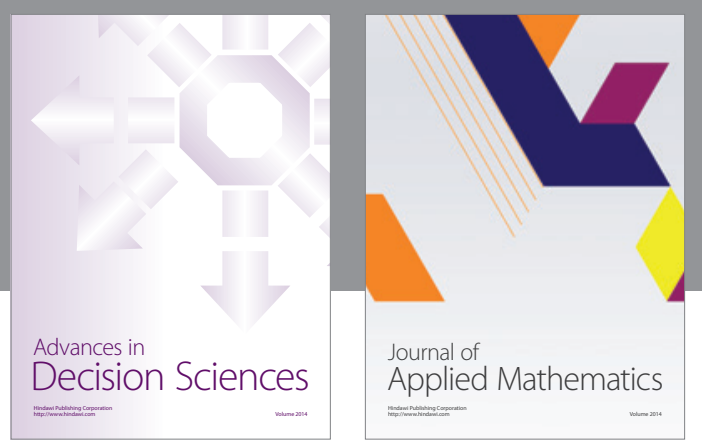

Algebra

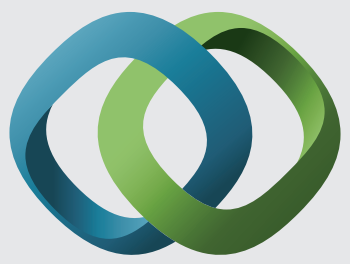

\section{Hindawi}

Submit your manuscripts at

http://www.hindawi.com
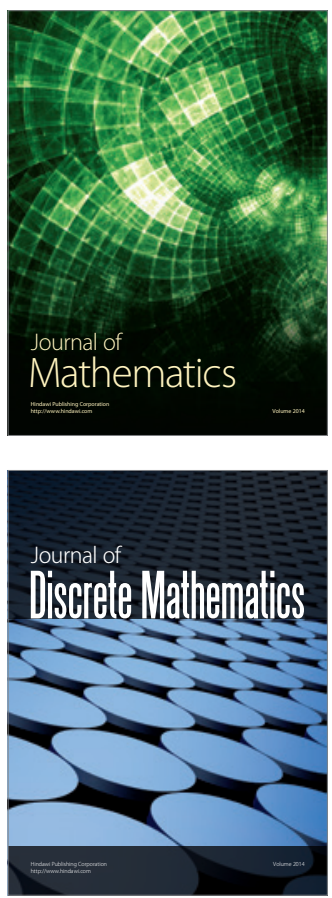

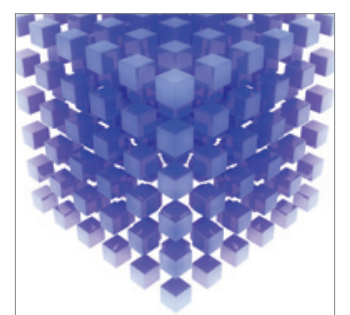

Mathematical Problems in Engineering
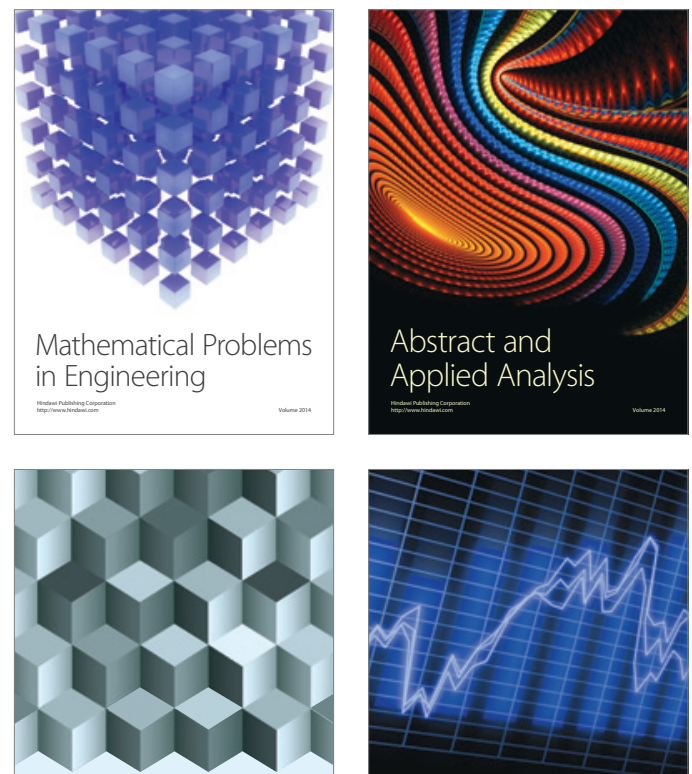

Journal of

Function Spaces

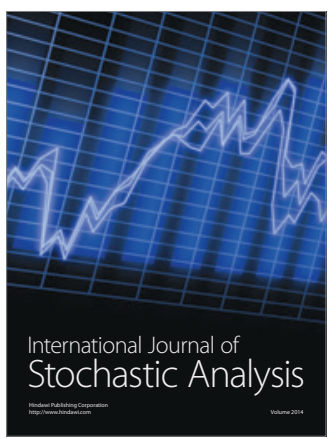

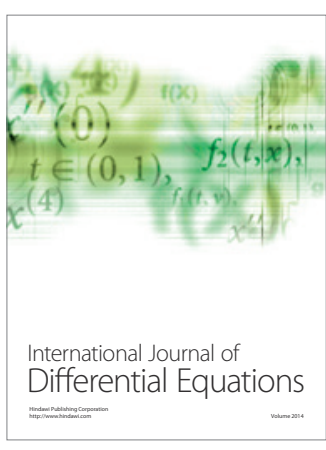
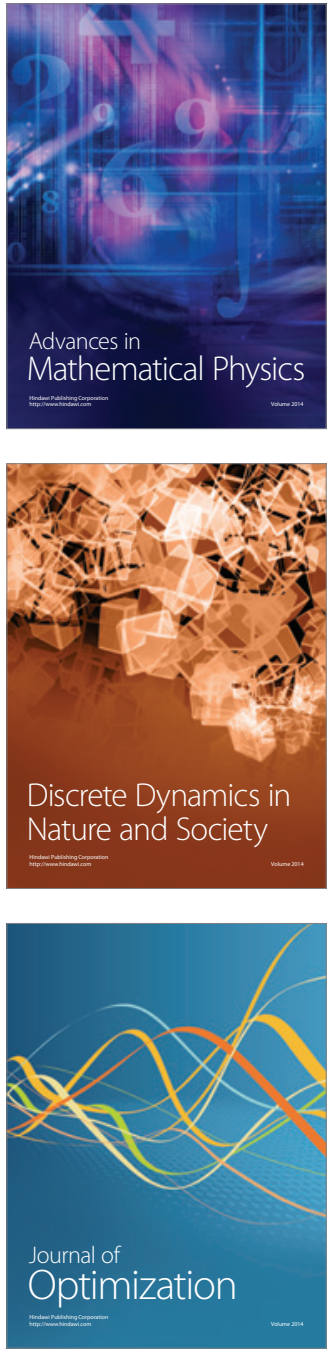